\title{
Zusammenhänge zwischen dem Verhalten des Körpergewichtes und dem immunologisch reagierenden Insulin im Serum alloxandiabetischer Ratten
}

\author{
B. RUDAS und W. WALDHÄUSL
}

Institut für allgemeine und experimentelle Pathologie der Universität Wien (Vorstand: Prof. Dr. A. LrNDNER) I. Medizinische Universitätsklinik (Vorstand: Prof. Dr. E. Deutscr)

Eingegangen am 12. Juli 1968

\begin{abstract}
Relationships between changes in body-weight and immunoreactive insulin in the serum of alloxan-diabetic rats

Summary. 7-9 weoks after alloxan administration it was possible to divide the diabetic rats into two groups: one with a gain in body weight (mean gain $60 \mathrm{~g}$ ) and the other with a loss of body weight (mean loss $28 \mathrm{~g}$ ). - In spite of this striking difference in body weight, there was no significant difference in the concentration of immunoreactive insulin of the serum (IRI) nor in the blood sugar levels between the two groups. - The results show that gain in body weight is possible even in chronically alloxandiabetic rats with marked hyperglycaemia and with a distinct diminution of IRI. - In the chronically diabetic rats with weight loss the relative weight of the adrenals was increased in comparison with the values found in normal rats or in the other group of alloxan-diabetic rats.
\end{abstract}

Relations entre le comportement du poids corporel et l'insuline immunoréactive dans le sérum de rats rendus diabétiques par l'alloxane

Résumé. 7-9 semaines après l'administration d'alloxane il y avait deux groupes de rats diabétiques; un qui avait gagné en moyenne $60 \mathrm{~g}$ et l'autre qui avait perdu en moyenne $28 \mathrm{~g}$. - Malgré cette différence de poids corporel, le taux d'insuline immunoréactive dans le sérum (IRI) et le taux du sucre dans le sang ne différaient pas significativement dans les deux groupes. - Ces résultats montrent, qu'une augmentation de poids corporel est possible chez les rats rendus chroniquement diabétiques par l'alloxane malgré une forte hyperglycémie et une diminution marquée d'IRI. - Chez les rats chroniquement diabétiques qui avaient perdu du poids corporel, le poids relatif des surrénales était plus élevé que chez les autres diabétiques ou chez les rats normaux.

Zusammenfassung. Alloxandiabetische Ratten wurden $7-10$ Wochen nach der Alloxangabe in eine Gruppe mit einer mittleren Gewichtszunahme von $60 \mathrm{~g}$ und in eine zweite mit einer Gewichtsabnahme von durchschnittlich 28 g geteilt. - Trotz der stark unterschiedlichen Gewichtsveränderung war kein statistisch erfaßbarer Unterschied bezüglich der Konzentration des IRI und des Blutzuckers zwischen den beiden Gruppen festzustellen. - Die Ergebnisse zeigen, daß auch bei chronisch alloxandiabetischen Ratten mit ausgeprägter Hyperglykämie und stark erniedrigten Serum-IRI eine Gewichtszunahme möglich ist. Bei den abgemagerten diabetischen Ratten war das relative Gewicht der Nebennieren höher als bei den diabetischen Ratten mit Gewichtszunahme bzw. bei den Kontrollen.

Key-words: Alloxan-diabetes in rat, weight gain, weight loss, blood sugar, immunoreactive insulin in serum, weight of the adrenals.
Das Vorkommen erhöhter Insulinkonzentrationen im Serum von übergewichtigen Personen und bei adipösen Diabetikern $[25,13,3,23,12,18,24] \mathrm{gab}$ Anla $B$ für tierexperimentelle Untersuchungen der Beziehung zwischen Fettsucht und Hyperinsulinismus. Eine Erhöhung des Seruminsulingehaltes konnte bei spontaner [4, 7], alimentärer und auch durch Schädigung des Hypothalamus induzierter Fettsucht beobachtet werden $[9,10]$. Der Zusammenhang zwischen Fettsucht und dem Insulingehalt im Serum erscheint jedoch noch nicht völlig geklärt. Während einerseits eine vermehrte Kalorienzufuhr und die Zunahme des Fettgewebes den Insulinbedarf erhöhen, kann andererseits ein erhöhter Insulingehalt des Blutes zur Hyperphagie führen und die Lipogenese verstärken. Auch wurde angenommen, daß eine Insulinresistenz der Muskulatur zur Hyperglykämie und damit zu vermehrter Insulinsekretion führen könnte, wobei gleichzeitig die Insulinempindlichkeit des Fettgewebes erhalten bleibt $[8,14,2,22]$.

In früheren Versuchen beobachteten wir [21], daß bei alloxandiabetischen Ratten einige Wochen nach der Alloxanapplikation - im sogenannten chronischen Stadium - sowohl eine starke Abmagerung, wie auch eine Gewichtszunahme eintreten kann. Es wurde angenommen, daß das verschiedene Ausmaß des Insulinmangels bei den einzelnen Tieren Ursache dieses unterschiedlichen Verhaltens sein könnte. Im folgenden wurde an einem Kollektiv chronisch alloxandiabetischer Ratten der Versuch unternommen, die Beziehungen zwischen Körpergewichtsveränderungen und dem Verhalten des immunologisch reagierenden Insulins (IRI) zu erfassen.

\section{Methodik}

Es wurden männliche Albinoratten eines SpragueDawley Stammes mit einem Gewicht von $180-220 \mathrm{~g}$ verwendet, die bei $22^{\circ} \mathrm{C}$ gehalten wurden. Die Tiere erhielten Rattenkeks ad libitum. Das Futter enthielt: Eiweiß $20.8 \%$, Fett $8.5 \%$ und Kohlehydrate $54.1 \%$ [15].

Die Alloxanapplikation erfolgte s.c. in einer Dosierung von $135 \mathrm{mg} / \mathrm{kg}$ Tiergewicht in $9 \%$ iger Lösung; 
diese wurde jeweils in isotoner Kochsalzlösung frisch zubereitet (Alloxan ,Loba-Chemie " $\mathrm{C}_{4} \mathrm{H}_{2} \mathrm{O}_{4} \mathrm{~N}_{2}+\mathrm{H}_{2} \mathrm{O}$ ). Es wurden jene Tiere als diabetisch betrachtet, die 48 Std nach der Alloxanapplikation eine Glucosurie über $2 \%$ (Tes-Tape Lilly) zeigten und im chronischen Stadium innerhalb von 24 Std mehr als $80 \mathrm{ml}$ Harn ausschieden und mindestens $20 \mathrm{~g}$ von den ad libitum angebotenen Rattenkeks verzehrten.

7-10 Wochen nach der Alloxangabe wurden die Ratten entsprechend der in dieser Zeit erfolgten Gewichtsveränderung in eine Gruppe mit Gewichtsabnahme und in eine zweite Gruppe mit Gewichtszunahme geteilt. In keiner zeitlichen Abhängigkeit von der Nahrungsaufnahme wurden die Tiere durch Entbluten getötet und der Zucker im Vollblut enzymatisch mit der Glucoseoxydase-Methode (im Test der Fa. C.F. Boehringer \& Soehne GmbH) bestimmt. Die Bestimmung des immunologisch reagierenden Insulins im Serum (IRI) erfolgte nach den Angaben von HaLES und RANDLE [11]. Als Bezugsgröße für die Festlegung der Eichkurve diente Humaninsulin (Novo-Kopenhagen). Weiterhin wurde das Gewicht der Nebennieren bestimmt. Als Vergleichsgruppen dienten stoffwechselgesunde Tiere und akut alloxandiabetische Ratten 48 Std nach der Alloxangabe. Die statistische Auswertung erfolgte mittels des $t$-Testes nach Student. Ein $p<0.05$ wurde als statistisch wahrscheinlich, ein $p<0.01$ als signifikant verschieden aufgefaßt.

\section{Ergebnisse}

Die Ergebnisse sind in der Tabelle angegeben.

Stoffwechselgesunde Ratten zeigten Blutzuckerwerte von $113 \pm 4.3 \mathrm{mg} \%$, die Konzentration des IRI war im Bereich von $54 \pm 3.5 \mu \mathrm{E} / \mathrm{ml}$. Das Nebennierengewicht betrug $42 \pm 3.0 \mathrm{mg}$.

Akut alloxandiabetische Ratten zeigten gegenüber der Kontrollgruppe signifikant erhöhte Blutzuckerwerte $(573 \mathrm{mg} \%)$ bei signifikant emiedrigter IRTKonzentration $(15 \pm 1.3 \mu \mathrm{E} / \mathrm{ml})$. Das mittlere Nebennierengewicht $(49 \pm 2.3 \mathrm{mg})$ war gegenüber jenem der Kontrollen etwas erhöht ( $p>0.05)$.

Sowohl bei den chronisch alloxandiabetischen Ratten mit einer mittleren Gewichtszunahme von $60 \pm$ $17 \mathrm{~g}$, wie auch bei denen mit einer mittleren Gewichtsabnahme von $28 \pm 6 \mathrm{~g}$ bestand eine starke Hyperglykämie (5i2 bzw. $553 \mathrm{mg} \%$ ). Die Werte des IRI waren signifikant niedriger $(23 \pm 2.7 \mathrm{bzw} .21 \pm 3.3 \mu \mathrm{E} / \mathrm{ml})$ als die gesunder Tiere. Gegenüber den Verhältnissen bei den akut diabetischen Ratten war der mittlere IRI-Gehalt des Serums bei den chronisch diabetischen Ratten mit Gewichtszunahme statistisch wahrscheinlich, bei den abgemagerten nicht signifikant erhöht.

Die mittleren Nebennierengewichte der ehronisch diabetischen Gruppen $(60 \pm 3.5 \mathrm{bzw} .63 \pm 5.0 \mathrm{mg})$ sind höher als die der Kontrolltiere $(p<0.01)$ und die der akut alloxandiabetischen Ratten $(p<0.05)$.

Zwischen den beiden Gruppen chronisch diabetischer Ratten bestanden neben der unterschiedlichen Gewichtsentwicklung keine signifikanten Unterschiede im Verhalten des Blutzuckers, des IRI oder des absoluten Nebennierengewichtes. Die Futteraufnahme der Tiere mit Gewichtszunahme war gegenüber jener der abgemagerten erhöht $(p<0.01)$.

Wenn wir die unterschiedliche Gewichtsentwicklung im chronisch alloxandiabetischen Stadium berücksichtigen, so fand sich bei den abgemagerten Tieren eine Erhöhung des relativen Gewichtes der Nebennieren $(p<0.01)$.

Tabelle 1. Die Tabelle zeigt die Blutzuckerwerte, Se-IRI-Konzentrationen und die Nebennierengewichte bei stoffwechselgesunden Kontrolltieren und bei alloxandiabetischen Ratten in verschiedenen Diabetesstadien; weiters die Futteraufnahme und die Gewichtsveränderung bei chronisch diabetischen Ratten

\begin{tabular}{|c|c|c|c|c|c|c|c|}
\hline Gruppe & $\begin{array}{l}\text { Veränderung } \\
\text { d. Körperge- } \\
\text { wichtes nach } \\
\text { der Alloxan- } \\
\text { applikation } \\
\text { g }(\mathbf{n})\end{array}$ & $\begin{array}{l}\text { Futterauf- } \\
\text { nahme in } \\
24 \text { Std } \\
g\end{array}$ & $\begin{array}{l}\text { Futterauf- } \\
\text { nahme in } \\
24 \mathrm{Std} \\
\text { g/100 g } \\
\text { Körperge- } \\
\text { wicht }\end{array}$ & $\begin{array}{l}\text { Blutzucker } \\
\text { mg\% }\end{array}$ & $\begin{array}{l}\text { Se-IRI } \\
\mu \mathrm{E} / \mathrm{ml}\end{array}$ & $\begin{array}{l}\text { Nebennieren- } \\
\text { gewicht } \\
\text { mg }\end{array}$ & $\begin{array}{l}\text { Nebennie- } \\
\text { rengewicht } \\
\text { mg/100 g } \\
\text { Körperge- } \\
\text { wicht }\end{array}$ \\
\hline $\begin{array}{l}\text { Stoffwechsel- } \\
\text { gesunde Tiere }\end{array}$ & - & - & - & $\begin{array}{l}113 \pm 4.6 \\
(5)\end{array}$ & $\frac{54}{(5)} \pm 3.5$ & $\begin{array}{l}42 \\
(7)\end{array}$ & $\begin{array}{l}19.65 \pm 2.0 \\
(7)\end{array}$ \\
\hline $\begin{array}{l}2 \text { Tage nach } \\
\text { Alloxan- } \\
\text { applikation }\end{array}$ & - & - & - & $\begin{array}{l}573 \pm 54 \\
(7)\end{array}$ & $\begin{array}{l}15 \\
(7)\end{array}$ & $\begin{array}{l}49 \pm 2.3 \\
(8)\end{array}$ & $\begin{array}{l}23.05 \pm 0.9 \\
(8)\end{array}$ \\
\hline $\begin{array}{l}7-10 \text { Wochen } \\
\text { nach Alloxanap- } \\
\text { plikation mit Ge- } \\
\text { wichtszunahme }\end{array}$ & $\underset{(8)}{+} 60 \pm 17$ & $\begin{array}{l}46 \\
(8)\end{array} \pm 2.5$ & $\begin{array}{l}16.9 \\
(8)\end{array}$ & $\begin{array}{l}512 \pm 25 \\
(8)\end{array}$ & $\begin{array}{l}23 \\
(8)\end{array} \pm 2.7$ & $\begin{array}{l}60 \\
(8)\end{array}$ & $\begin{array}{l}21.92 \pm 1.4 \\
(8)\end{array}$ \\
\hline $\begin{array}{l}7-10 \text { Wochen } \\
\text { nach Alloxanap- } \\
\text { plikation mit Ge- } \\
\text { wichtsabnahme }\end{array}$ & $\frac{-28}{(10)} \pm 6$ & $\begin{array}{l}37 \pm 2.7 \\
(10)\end{array}$ & $\begin{array}{l}19.3 \pm 1.2 \\
(10)\end{array}$ & $\begin{array}{l}553 \pm 33 \\
(10)\end{array}$ & $\begin{array}{l}21 \pm 3.3 \\
(10)\end{array}$ & $\begin{array}{l}63 \pm 5.0 \\
(10)\end{array}$ & $\begin{array}{l}33.5 \\
(10)\end{array}$ \\
\hline
\end{tabular}

Die Zahlenangaben betreffen Mittelwerte \pm mittlere Fehler der Mittelwerte und in Klammer die Tierzahl 


\section{Diskussion}

Der IRI-Gehalt im Serum alloxandiabetischer Ratten wurde von Morgan und Lazarow [19, 20] untersucht. Sie fanden bei Tieren mit ausgeprägter Hyperglykämie niedrigere Insulinwerte als bei den sogenannten subdiabetischen, mit normalen Blutzuckerwerten aber verminderter Glucosetoleranz.

Wir ermittelten bei den 48 Std nach Alloxangabe untersuchten Ratten niedrigere IRI-Werte als bei denen im chronischen Stadium. Bei unseren Versuchsbedingungen lag jedoch der Blutzuckerwert auch bei den chronisch diabetischen Tieren über $500 \mathrm{mg} \%$. Die relative Erniedrigung des Blutzuckerspiegels und Erhöhung des IRI-Gehaltes im chronischen Stadium könnte auf eine partielle Regeneration des $\beta$-Zellapparates zurückgeführt werden. Zur Bestätigung dieser Vermutung wären allerdings wiederholte Bestimmungen des IRI bei den einzelnen Tieren zu verschiedenen Zeitpunkten nach der Alloxanapplikation notwendig. Höhere IRI-Werte sind allerdings keine Voraussetzung: für das Erreichen des chronischen Stadiums, da die IRI-Konzentration akut alloxandiabetischer Ratten zwischen $10-20 \mu \mathrm{E} / \mathrm{ml}$ gelegen war, dieser Bereich jedoch nur von 8 der untersuchten 18 chronisch diabetischen Tiere überschritten wurde.

Der Vergleich zwischen den beiden chronisch diabetischen Gruppen zeigt, daß bei den abgemagerten Ratten der Blutzucker und die Nebennierengewichte etwas höher und der IRI-Gehalt etwas niedriger war als bei den Tieren mit Gewichtszunahme. Weiters waren in der Gruppe mit Gewichtszunahme mehr Tiere mit höheren IRI-Werten zu finden. Das Zusammentreffen dieser Befunde könnte den Eindruck erwecken, da $B$ bei den abgemagerten chronisch diabetischen Ratten der Diabetes stärker ausgeprägt war. Doch sind die erwähnten Unterschiede gering und nicht signifikant. Die Einzelwerte des IRI waren bei den abgemagerten Ratten zwischen $11-45 \mu \mathrm{E} / \mathrm{ml}$ und bei den Tieren mit Gewichtszunahme zwischen 14-35 $\mu \mathrm{E} / \mathrm{ml}$ gelegen. Eine Korrelation zwischen dem Verhalten von IRI, Blutzucker und der Entwicklung des Körpergewichtes konnte somit nicht festgestellt werden.

In der vorliegenden Untersuchung wurde gezeigt, daß eine Gewichtszunahme bei chronisch alloxandiabetischen Ratten auch bei erniedrigtem Serum-IRIGehalt erreicht werden kann. In diesem Zusammenhang erscheinen Untersuchungen von MaLaIsse [17] und LEMONNIER [16] erwähnenswert, die erstmals bei Ratten durch Verfütterung fettreicher Nahrung eine Fettsucht mit erniedrigter Insulinsekretion hervorrufen konnten. Diese Befunde zeigten, daß eine Erhöhung der Insulinausschüttung keine unbedingte Voraussetzung für das Zustandekommen der Fettsucht darstellt.

Im Gegensatz zu manchen Literaturstellen $[5,6]$ fanden wir, daß die durch Alloxaninjektion bedingte Hypertrophie der Nebennieren erst nach mehrwöchi- ger Diabetesdauer beträchtliche Ausmaße erreicht. In diesem Zusammenhang soll darauf hingewiesen werden, daß die Reaktion der Nebennieren vielfach von der Alloxanmenge, vom Grad der folgenden Stoffwechselstörung und von dem verwendeten Rattenstamm abhängig ist [1].

\section{Literatur}

1. Appregarth, A.: Histochemical changes in the adrenal cortex of the rat in alloxan diabetes. Endocrinology 44, 197-199 (1949).

2. BJorntorp, P., B. Larssox, W. Szostak, T. SoherSTEN, and L. SJostrom: Labelling of adipose tissue triglycerides with $\mathrm{C}^{14}$-glucose in man. Abstracts from the Second Annual Meeting of the European Society of Clinical Investigation. p. 11 (1968).

3. Botterman, P., K. Schwarz und K. Kopetz: Über das Verhalten der insulinähnlichen Activität im Serum bei der Fettsucht. Dtsch. med. Wschr. 90, 917-921 (1965).

4. Christophe, J., Y. Dagmais, and J. Mayer: Increased circulating insulinlike activity in obese-hyperglycaemic mice. Nature 184, 61-62 (1959).

5. Devecerskr, M.S., and T.F. Frawley: Adrenal steroid production in rats with alloxan diabetes. Endocrinology 73, 386-391 (1963).

6. FrELD, J.B.: Prevention of adrenal cortical hypertrophy in diabetic rats by the use of insulin. Eindocrinology 56, $499-506$ (1955).

7. Gonet, A.E., W. Stauffacher, R. Pictert, and A.E. Renold: Obesity and Diabetes Mellitus with Striking Congenital Hyperplasia of the Islets of Langerhans in Spiny Mice. Diabetologia 1, 162-172 (1966).

8. Hacket, D.B., H.E. Lebovitz, L.A. Frohman, E. Mikat, and K. Schmidt-Nielsen: Effect of caloric restriction on the glucose tolerance and plasma insulin of the sand rat. Metabolism 16, 1133-1140 (1967).

9. - L. Frohman, E. Mirkat, H. E. Lebovitz, K. SCHMIDT-NIELSEN, and T.D. KINNEY: Effect of diet on the glucose tolerance and plasma insulin levels of the sand rat. Diabetes 15, 105-114 (1966).

10. Hales, C.N., and G.C. Kennedy: Plasma glucose, non-esterified fatty acid and insulin concentrations in hypothalamic-hyperphagic rats. Biochem. J. 90, $620-624$ (1964).

11. - , and P.J. RANDLE: Immunoassay of insulin with insulin antibody precipitate. Biochem. J. 88, 137-146 (1963).

12. JahNKe, K., H. DaWEKe, W. SchtLLING, R. RÜENAUVER, and K. OBERDISSE: Der potentielle Diabetes. S. 57-75. 2. Tagung der Deutschen Diabetes-Gesellschaft Wiesbaden (April 1966). Berlin-HeidelbergNew York: Springer 1967.

13. Karam, J.H., G.M. Grodsky, and P.H. Forsham: Excessive insulin response to glucose in obese subjects as measured by immunochemical assay. Diabetes 12, 197-204 (1963).

14. - - F. Ch. Pavlatcos, and P.H. Forsham: Critical factors in excessive serum insulin response to glucose. Lancet 1965 I, 286-290.

15. LEMBECK, F.: Die Wartung und Fütterung von Ratten im Laboratorium. Arzneimittel-Forsch. 3, 50-52 (1953).

16. LEMONNIER, D.: Characteristic of rat adipose tissue and adrenal cortex in experimental obesity produced by a high fat diet. Third International Meeting of Endocrinology (Marseilles, May 1968).

17. Matatsse, W.: Islet function in experimental obesity. Third International Meeting of Eindocrinology (Marseilles, May 1968). 
18. Mmuant, F., J. Lawedri, K.M. Barteit u. E.F. PHEIfTHR : Immunologisch meßbares Insulin boi Stoffwechselgesunden, Fettsüchtigen und adipösen Diabetikern nach intravenöser Gabe von Glucose, Tolbutamid und Glueagon. Diabetologia 3, 422-427 (1967).

19. Morgan, C.R., and A. Lazarow: Immounoassay of insulin: two antibody system. Plasma insulin levels of normal, subdiabetic and diabetic rats. Diabetes 12, $115-126(1963)$.

20. - - Immunoassay of pancreatic and plasma insulin following alloxan injection of rats. Diabetes 14, $669-$ 671 (1965).

21. Rudus, B.: Das Verhalten der Serumlipide in verschiedenen Diabetesstadien der Ratte. Wien. klin. Wschr. 79, 377-382 (1967).

22. StadFFACHER, W., A.E. Lambert, D. VECChIo, and A. E. RFNoLD: Measurements of insulin activities in pancreas and serum of mice with spontaneous and induced obesity and hyperglycemia, with considerations on the pathogenesis of the spontaneous syndrome. Diabetologia 3, 230-237 (1967).

23. VAGUE, J., J.L. CODACCIONI, J. Boyer, and $\mathrm{Ph}$. VAGUE: Relationship between plasma insulin and cortisol production rate in diabetic and non diabetic obese subjects. Sixth Internat. Diabetes Congress Stockholm. Abstract Excerpta med. 140, 165 (1967).

24. WALDHÄUSL, W., u. E.G. HUBER: Immunologisch reagierendes Insulin bei adipösen Kindern. Z. Kinderheilk. 104, 128-134 (1968)

25. YAtOW, R. S., and S.A. Berson: Plasma insulin concentrations in nondiabetic and early diabetic subjects. Diabetes 9, 254-260 (1960).

Univ.-Doz. Dr. Barbara RUdAS

Institut für allgemeine und experimentelle Pathologie der Universität Wien A-1090 Wien Währinger Straße 13 